Draft VERSiOn MAY 15, 2018

Preprint typeset using $\mathrm{LAT}_{\mathrm{E}} \mathrm{X}$ style emulateapj v. 5/2/11

\title{
PROSPECTS FOR CONSTRAINING THE SPIN OF THE MASSIVE BLACK HOLE AT THE GALACTIC CENTER VIA THE RELATIVISTIC MOTION OF A SURROUNDING STAR
}

\author{
Qinguuan $\mathrm{Yu}^{1, \dagger}$, Fupeng Zhang ${ }^{1,3}$, And Youjun Lu ${ }^{2,4}$ \\ ${ }^{1}$ Kavli Institute for Astronomy and Astrophysics, Peking University, Beijing, 100871, China; ${ }^{\dagger}$ yuqj@pku.edu.cn \\ ${ }^{2}$ National Astronomical Observatories, Chinese Academy of Sciences, Beijing, 100012, China; luyj@nao.cas.cn \\ ${ }^{3}$ School of Physics and Astronomy, Sun Yat-Sen University, Guangzhou 510275, China; zhangfp7@mail.sysu.edu.cn \\ ${ }^{4}$ College of Astronomy and Space Sciences, University of Chinese Academy of Sciences, Beijing, 100049, China \\ Draft version May 15, 2018
}

\section{ABSTRACT}

In this paper, we investigate the quality of constraining the spin of the massive black hole (MBH) at the Galactic center (GC) by using full general relativistic simulations of the motion of a surrounding star. We obtain the dependence mapping of the spin-induced signals on any spin direction of the $\mathrm{MBH}$ for given example stars, which indicates the feasibility to test whether the spin direction is the same as the normal of the young stellar disk located at the GC, and, further to provide insights into the assembly history of the MBH. We demonstrate the quality of constraining the MBH spin that may be achieved, given any set of the astrometric and the redshift precisions of observational facilities. We find that in the ranges of the astrometric and the velocity precisions with 1-30 $\mu$ as and $0.1-10 \mathrm{~km} \mathrm{~s}^{-1}$, an improvement in astrometric precision would be more effective at improving the quality of constraining the spin than an improvement in velocity precision. We obtain the parameter space of the semimajor axis and the eccentricity for the orbit of the target star that a high-precision constraint on the GC MBH spin can be obtained via the motion of the star. Our results show that the spin of the GC MBH can be constrained with $1-\sigma$ error $\lesssim 0.1$ or even $\lesssim 0.02$ by monitoring the orbital motion of a star, if existing as expected, with semimajor axis $\lesssim 300 \mathrm{AU}$ and eccentricity $\gtrsim 0.95$ over a period shorter than a decade through future facilities.

Subject headings: Black hole-physics - gravitation - Galaxy: center - relativistic processes

\section{INTRODUCTION}

Astronomical black holes (BHs) are believed to be described by the Kerr metric with only two parameters, i.e., mass and spin (e.g., Kerr 1963). Observations have exclusively demonstrated the existence of a massive $\mathrm{BH}$ $(\mathrm{MBH})$, with mass $\sim 4 \times 10^{6} M_{\odot}$, in the Galactic center (GC; Ghez et al.|2008; Gillessen et al.|2009). However, it is still under inspection on whether or not such an object is a Kerr BH.

Accurately monitoring the motion of a star close to the GC $\mathrm{MBH}$ has long been anticipated to provide strong dynamical constraints on the $\mathrm{MBH}$ spin and metric (Jaroszvnski 1998; Fragile \& Mathews 2000; Rubilar \& Eckart 2001; Zucker et al. 2006; Will 2008; Kannan \& Saha 2009; Angélil \& Saha 2010; Angélil et al. 2010; Angélil \& Saha 2011; Merritt et al. 2010; Sadeghian \& Will 2011; Iorio 2011). Outstanding questions related to this endeavor are as follows. (1) How accurate do the astrometric and velocity/redshift measurements need to be to provide a tight constraint on the MBH spin by using the motion of a star, e.g., $\mathrm{S} 2 / \mathrm{S} 0-2$, the closest one to the MBH currently detected (Ghez et al. 2008; Gillessen et al. 2009)? (2) What is the parameter space for a possibly existing star within the orbit of S2/S0-2 that may help to put a strong constraint on the MBH spin and metric with the next-generation (or future) facilities? Constraining the metric and the spin of the GC MBH is of fundamental importance for testing general relativity (GR), and a constraint on the spin direction would also provide insights into the assembly history of the MBH In this paper, we address the above two questions and obtain guiding maps for future observational endeavors on constraining the $\mathrm{GC} \mathrm{MBH}$ spin via extensive numerical simulations of the relativistic motion of a surrounding star in a large parameter space. We adopt the full GR numerical method developed in Zhang et al. (2015) to investigate the spin-induced relativistic effects on the trajectory and the redshift of a star measured by a distant observer. As an alternative approach to those previous ones that utilize the perturbative (post-Newtonian or weak-field) approximations (e.g., Will 2008; Angélil et al. 2010; Angélil \& Saha 2010), the full GR method is efficient and accurate in obtaining the orbital motion of a target star rotating around the GC $\mathrm{MBH}$ and the photon propagation from the star to the observer.

Whether or not those spin-induced effects can be measured and a dynamical constraint on the GC MBH spin can be made depends on how precise the position and velocity measurements that current and future facilities, such as the GRAVITY on the Very Large Telescope Interferometer (VLTI), the Thirty Meter Telescope (TMT), and the European Extremely Large Telescope (E-ELT), can achieve. For example, for the TMT, the position

\footnotetext{
1 The spin direction may help to reveal the assembly history of the GC MBH, which can be understood as follows. If the $\mathrm{MBH}$ spun up by continuous disk accretion with the same orientation as that of the young stellar disk at the GC, the spin direction should align with the normal of the disk; and if the MBH grew up via many episodes of disk accretion with random orientations, then the spin value may be small and the spin direction may be significantly different from that of the normal of the young stellar disk (as inferred from King \& Pringle 2006).
} 
precision could reach $10 \mu$ as for bright stars with K-band magnitude $m_{K}<15$; while it could be $>50 \mu$ as for those stars with $m_{K}>16$ because of 'source confusion' (Yelda et al. 2013). For the GRAVITY, the position precision is expected to reach $\sim 10 \mu$ as for bright stars with $m_{K} \lesssim 16.3$; while it could be $\sim 200 \mu$ as for stars fainter than $m_{K} \sim 18.8$ (Stone et al. 2012).

The paper is organized as follows. We describe the motion equations in the Kerr metric in Section 2. We introduce the geometry and the coordinate systems for the system of a star rotating around an $\mathrm{MBH}$ in Section 3. Our full GR method is summarized in Section 4 for calculating the relativistic motion of the star and the photon propagation from the star to the observer $2 \mathrm{We}$ numerically generate mock observations for stars rotating around the $\mathrm{GC} \mathrm{MBH}$, covering a large parameter space of stellar orbits as well as the position and the redshift precisions. With those mock observations, we illustrate how the GC MBH spin and its direction can be simultaneously constrained by using the Markov Chain Monte Carlo (MCMC) fitting technique. We present our results on the quality of the possible spin constraints by future facilities in Section [5. A summary and discussion are given in Section 6 .

\section{MOTION EQUATIONS IN A KERR METRIC}

In the Boyer-Lindquist (BL) coordinates $(t, r, \theta, \phi)$ (Boyer \& Lindquist 1967) for the Kerr metric, the motion of a particle (hereafter, a star or a photon) is given by

$$
\begin{aligned}
& \Sigma \frac{d r}{d \tau}= \pm \sqrt{R} \\
& \Sigma \frac{d \theta}{d \tau}= \pm \sqrt{\Theta} \\
& \Sigma \frac{d \phi}{d \tau}=-a+\frac{\lambda}{\sin ^{2} \theta}+\frac{a T}{\Delta}, \\
& \Sigma \frac{d t}{d \tau}=-a^{2} \sin ^{2} \theta+a \lambda+\frac{\left(r^{2}+a^{2}\right) T}{\Delta}
\end{aligned}
$$

with

$$
\begin{aligned}
\Sigma= & r^{2}+a^{2} \cos ^{2} \theta, \\
\Delta= & r^{2}-2 r+a^{2}, \\
T= & r^{2}+a^{2}-\lambda a, \\
R= & \left(1-\xi^{2}\right) r^{4}+2 \xi^{2} r^{3}+\left[a^{2}\left(1-\xi^{2}\right)-q^{2}\right. \\
& \left.-\lambda^{2}\right] r^{2}+2\left[(a-\lambda)^{2}+q^{2}\right] r-a^{2} q^{2}, \\
\Theta= & q^{2}-\left[a^{2}\left(\xi^{2}-1\right)+\frac{\lambda^{2}}{\sin ^{2} \theta}\right] \cos ^{2} \theta .
\end{aligned}
$$

Here $\tau$ is an affine parameter, $a$ is the dimensionless spin parameter, $\xi=m / E, \lambda=L_{z} / E$, and $q^{2}=$ $Q / E^{2}$ (Chandrasekhan 1983). The four motion constants of the particle are its rest mass $(m)$, energy at infinity $(E)$, azimuthal angular momentum $\left(L_{z}\right)$, and Carter's constant $(Q)$ (see eq. 182 in Chapter 7 in Chandrasekhar 1983). For simplicity, the natural units are adopted

2 The photon propagation effects are important for accurately measuring the MBH spin as pointed out by Angélil \& Saha (2010), which are also shown in Figures 2 and 3 and discussed in Section 5.2 of this paper below.

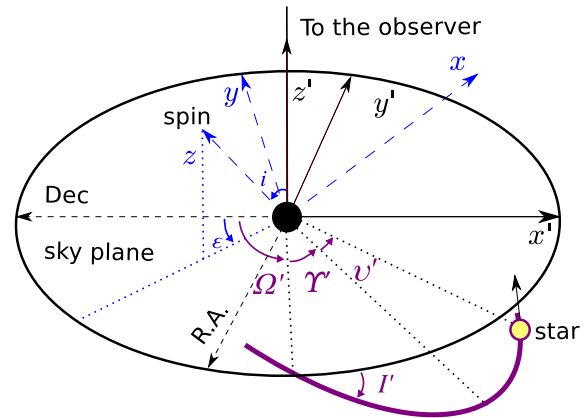

FIG. 1. - Schematic diagram for a star rotating around the GC MBH. A pseudo-Cartesian coordinate system $\left(x^{\prime}, y^{\prime}, z^{\prime}\right)$ is defined in the distant observer's rest frame $(\mathrm{ORF})$, originating at the $\mathrm{MBH}$, with $\vec{x}^{\prime}$ representing the reference direction on the sky plane $x^{\prime} y^{\prime}$, and $\vec{z}^{\prime}$ being the line of sight. The directions of the declination and the right ascension are $-\vec{x}^{\prime}$ and $-\vec{y}^{\prime}$. Another pseudo-Cartesian coordinate system $(x, y, z)$ is in the $\mathrm{MBH}$ frame, defined with its origin at the $\mathrm{MBH}, \vec{z}$ representing the spin direction of the $\mathrm{MBH}$, and $\vec{y}$ being the line of intersection of the MBH equatorial plane with the $x^{\prime} y^{\prime}$ plane. The spin direction is described by two angles, i.e., $i$ between $\vec{z}$ and $\vec{z}^{\prime}$, and $\epsilon$ between the projection vector of $\vec{x}$ on the $x^{\prime} y^{\prime}$ plane and $\vec{x}^{\prime}$. The stellar orbit is approximated as a Newtonian ellipse in the ORF, determined by its six orbital elements: semimajor axis $\left(a_{\mathrm{orb}}\right)$, eccentricity $\left(e_{\mathrm{orb}}\right)$, longitude of ascending node $\left(\Omega^{\prime}\right)$, position angle of periapsis $\left(\Upsilon^{\prime}\right)$, true anomaly $\left(v^{\prime}\right)$, and inclination $\left(I^{\prime}\right)$.

above, i.e., the gravitational constant $G$, the speed of light $c$, and the $\mathrm{MBH}$ mass $M_{\bullet}$ are all set to one.

\section{GEOMETRY OF THE STAR-MBH SYSTEM}

Figure 1 illustrates the geometry of the system composed of a star rotating around the $\mathrm{GC} \mathrm{MBH}$ with mass M. and distance of $R_{\mathrm{GC}}$ to the sun. We define two pseudo-Cartesian coordinate systems, i.e., $(x, y, z)$ and $\left(x^{\prime}, y^{\prime}, z^{\prime}\right)$. The former is in the MBH frame with $\vec{z}$ representing the spin direction, and the latter is in the observer's rest frame (ORF). As seen from the figure, the $(x, y, z)$ frame can be obtained by rotating the $\left(x^{\prime}, y^{\prime}, z^{\prime}\right)$ frame first around the $y^{\prime}$-axis clockwisely by an angle of $i$ and then around the $z^{\prime}$-axis counter-clockwisely by an angle of $\epsilon$. For a star rotating around an $\mathrm{MBH}$ with a semimajor axis much larger than $r_{\mathrm{g}} \equiv G M_{\bullet} / c^{2}$, its orbit in the ORF can be instantaneously approximated as a Newtonian ellipse, and its initial position and velocity are determined by its six orbital elements $\left(a_{\text {orb }}, e_{\text {orb }}, I^{\prime}, \Omega^{\prime}, \Upsilon^{\prime}, v^{\prime}\right) 3$

The initial position of the star in the local nonrotating rest frame (LNRF) with $(x, y, z)$ coordinates at time $t_{\star, 0}$ can be converted to the BL coordinates $\left(t_{\star, 0}, r_{\star, 0}, \theta_{\star, 0}, \phi_{\star, 0}\right)$; and the initial tetrad velocity $\mathbf{u}_{\star, 0}=$ $\left(u_{\star, 0}^{t},, u_{\star, 0}^{r}, u_{\star, 0}^{\theta}, u_{\star, 0}^{\phi}\right)$ of the star in the BL coordinates can be obtained from the three-velocity $\mathbf{v}_{\star, 0}=$ $\left(v_{\star, 0}^{r}, v_{\star, 0}^{\theta}, v_{\star, 0}^{\phi}\right)$ in the LNRF (see Chandrasekhar 1983). The motion constants $\lambda, q$, and $\xi$ can also be obtained from the position and the tetrad velocity of the star.

\section{FULL GENERAL RELATIVISTIC NUMERICAL METHOD}

We use the full GR numerical method developed in Zhang et al. (2015) to calculate both the stellar motion around the $\mathrm{GC} \mathrm{MBH}$ and the photon propagation from the star to the observer.

3 The position angle of periapsis $\Upsilon^{\prime}$ is related to the argument of periapsis (denoted by $\omega^{\prime}$ ) by $\omega^{\prime}=\pi+\Upsilon^{\prime}$. The changes of the two angles are the same, i.e., $\delta \omega^{\prime}=\delta \Upsilon^{\prime}$. 
For a star rotating around an $\mathrm{MBH}$ with any set of $\left(\xi, \lambda, q^{2}\right)$, its orbital evolution can be obtained by numerically solving Equations (1)-(4). We adopt the explicit fifth (fourth)-order Runge-Kutta method (Hairer et al. $1993)$ to integrate these equations, and we set high relative accuracies $\left(\leq 10^{-12}\right)$ for those integral quantities in order to calculate the spin-induced effects precisely.

For a photon from the star received by a distant observer located at $(r, \theta, \phi)=\left(R_{\mathrm{GC}}, i, 0\right)$, its trajectory may be bended and its energy at the receiving time may be shifted away from that at the emission time. The position of the star on the observer's sky plane can be described by impact parameters $\alpha$ and $\beta$, with the former representing the displacement of the star image perpendicular to the projected symmetry axis of the $\mathrm{MBH}$ and the latter representing the displacement parallel to this axis. The motion constants are $\xi=0$, $\lambda=-\alpha \sin i$, and $q^{2}=\beta^{2}+\left(\alpha^{2}-a^{2}\right) \cos ^{2} i$ for a photon received by the observer at $(\alpha, \beta)$ (Chandrasekhar 1983). We adopt the ray-tracing technique (Rauch \& Blandford 1994, Lu \& Yu 2001, for details see Zhang et al. 2015) to search the parameter space $(\alpha, \beta)$ for those photons received by the observer and emitted from a star on a relativistic orbit. As a result, the position of the star on the sky plane $\left(\alpha_{k}, \beta_{k}\right)$ at a given observing time $t_{\mathrm{obs}, k}$ is connected to the position of the star at $\left(t_{k}, r_{k}, \theta_{k}, \phi_{k}\right)$. In the mean time, the tetrad-momentum of the photon $\mathbf{p}_{k}$ can also be obtained, and the redshift (measured through the shift of emission or absorption lines) $Z_{k}=-\mathbf{p}_{k} \cdot \mathbf{u}_{\star, k} / E_{0}-1$, where $E_{0}$ is the energy of the photon in the rest frame of the star at the emission time, $\mathbf{u}_{\star, k}$ is the tetrad velocity of the target star at time $t_{\mathrm{obs}, k}$. Therefore, the observational quantities of the star (position trajectory and redshift curve) can be mapped to its relativistic orbital motion.

For the full GR numerical calculations below, we set the numerical accuracies for positions and redshifts (or velocities) to be $10^{-4} \mu$ as and $10^{-6} \mathrm{~km} \mathrm{~s}^{-1}$, respectively.

\section{RESULTS}

\subsection{Numerical results from full GR calculations}

We show that the spin-induced effects on the motion of a given star are sensitive to the spin direction in Figures 2 and 3. where the spin-induced signals are obtained by the difference of the signals between the cases of the $\mathrm{MBH}$ spin $a=0.99$ and $a=0$.

Figure 2 shows the dependence of the spin-induced signals on the spin directions for $\mathrm{S} 2 / \mathrm{S} 0-2$ after the motion of one full orbit, where the initial orbital elements of the star are set to $a_{\text {orb }}=984 \mathrm{AU}, e_{\text {orb }}=0.88, I^{\prime}=135^{\circ}$, $\Omega^{\prime}=225^{\circ}$, and $\Upsilon^{\prime}=63^{\circ}$ Gillessen et al. 2009). The left panel of Figure 2 shows the position displacements at the apoapsis of $\mathrm{S} 2 / \mathrm{S} 0-2$ between the case with a rapidly rotating $\mathrm{MBH}(a=0.99)$ and that with a non-rotating $\mathrm{MBH}$; and the middle panel shows the maximum value of the spin-induced redshift differences over one full orbital period between these two cases. As seen from the left panel, the spin-induced position displacement at apoapsis is most significant, i.e., $\sim 13 \mu$ as for $a=0.99$ when $(i, \epsilon)=\left(49^{\circ}, 125^{\circ}\right)$ or $\left(131^{\circ}, 305^{\circ}\right)$, and the least significant, i.e., $\sim 0.2 \mu$ as for $a=0.99$ when $(i, \epsilon)=\left(50^{\circ}, 351^{\circ}\right)$ or $\left(130^{\circ}, 171^{\circ}\right)$. As seen from the middle panel, the maximum value of the spin-induced redshift differences over a full orbit is the most significant, i.e., $0.56 \mathrm{~km} \mathrm{~s}^{-1}$ for $a=0.99$ when $(i, \epsilon)=\left(28^{\circ}, 127^{\circ}\right)$ or $\left(152^{\circ}, 307^{\circ}\right)$, and the least significant, i.e., $\sim 10^{-2} \mathrm{~km} \mathrm{~s}^{-1}$ if $(i, \epsilon)=$ $\left(118^{\circ}, 144^{\circ}\right)$ or $\left(72^{\circ}, 324^{\circ}\right)$. As seen from the figure, the normal of the clockwise rotating stellar disk is close to the direction of the spin with the maximum spin-induced signals. If the spin direction is the same as the normal of the clockwise rotating stellar disk, the spin-induced apparent position displacement at the apoapsis and the maximum redshift difference are close to the above maximum values (see Fig. 2); and accurate measurements of the motion of the star are helpful to test whether the two directions are the same or significantly different.

One may note here that constraining the spin direction is also important as it may record the assembly history of the $\mathrm{MBH}$. For example, if the spin direction is the same as the normal of the young stellar disk in the GC, it may indicate that the growth of the $\mathrm{GC} \mathrm{MBH}$ via the previously existing gas disk, which coincides with the stellar disk, is important; or if the spin direction is significantly different from the normal of the stellar disk, which might suggest that the accretion episodes that happened at a later time are chaotic and the growth of the $\mathrm{MBH}$ via a single accretion episode is not significant.

Similar to Figure 2 Figure 3 shows the dependence of spin-induced signals on the spin directions for a hypothetical star after the motion of one full orbit by assuming the initial orbital elements $a_{\text {orb }}=300 \mathrm{AU}$, $e_{\mathrm{orb}}=0.95, I^{\prime}=151^{\circ}, \Omega^{\prime}=175^{\circ}$, and $\Upsilon^{\prime}=185^{\circ}$. The orbital orientation of this star is set to be the same as that of S0-102 (Mever et al. 2012). The orbital period of such a star is $\sim 2.6 \mathrm{yr}$. As seen from the left panel of the figure, the spin-induced position displacement at apoapsis is most significant, i.e., $\sim 84.9 \mu$ as for $a=0.99$ when $(i, \epsilon)=\left(42^{\circ}, 78^{\circ}\right)$ or $\left(138^{\circ}, 258^{\circ}\right)$, and the least significant, i.e., $\sim 1.7 \mu$ as for $a=0.99$ when $(i, \epsilon)=$ $\left(116^{\circ}, 140^{\circ}\right)$ or $\left(64^{\circ}, 320^{\circ}\right)$. As seen from the middle panel, the maximum value of the spin-induced redshift differences over a full orbit is the most significant, i.e., $38.9 \mathrm{~km} \mathrm{~s}^{-1}$ for $a=0.99$ when $(i, \epsilon)=\left(27^{\circ}, 47^{\circ}\right)$ or $\left(153^{\circ}, 227^{\circ}\right)$, and the least significant, i.e., $\sim 1.3 \mathrm{~km} \mathrm{~s}^{-1}$ if $(i, \epsilon)=\left(101^{\circ}, 125^{\circ}\right)$ or $\left(79^{\circ}, 305^{\circ}\right)$. If the spin direction is the same as the normal of the clockwise rotating stellar disk, the spin-induced apparent position displacement at the apoapsis is about one third of the above maximum value, and the spin-induced redshift difference is about half of the above maximum value.

\subsection{Comparison with analytical approximations} 5.2.1. Analytical approximations

Orbital precession: the leading spin effect on the motion of a star is the Lense-Thirring (LT) precession of the star's orbital plane, which causes the changes of the longitude of ascending node $(\Omega)$ and the position angle of periapsis $(\Upsilon=\omega-\pi)$ per orbit by

$$
\delta \Omega_{\mathrm{LT}}=4 \pi a a_{\mathrm{orb}}^{-3 / 2}\left(1-e_{\mathrm{orb}}^{2}\right)^{-3 / 2}
$$

and

$$
\delta \Upsilon_{\mathrm{LT}}=-3 \delta \Omega_{\mathrm{LT}} \cos \zeta
$$

respectively (e.g., Lense \& Thirring Mashhoon et al. 1984; Wex \& Kopeikin 1999). Here $\Omega$ 


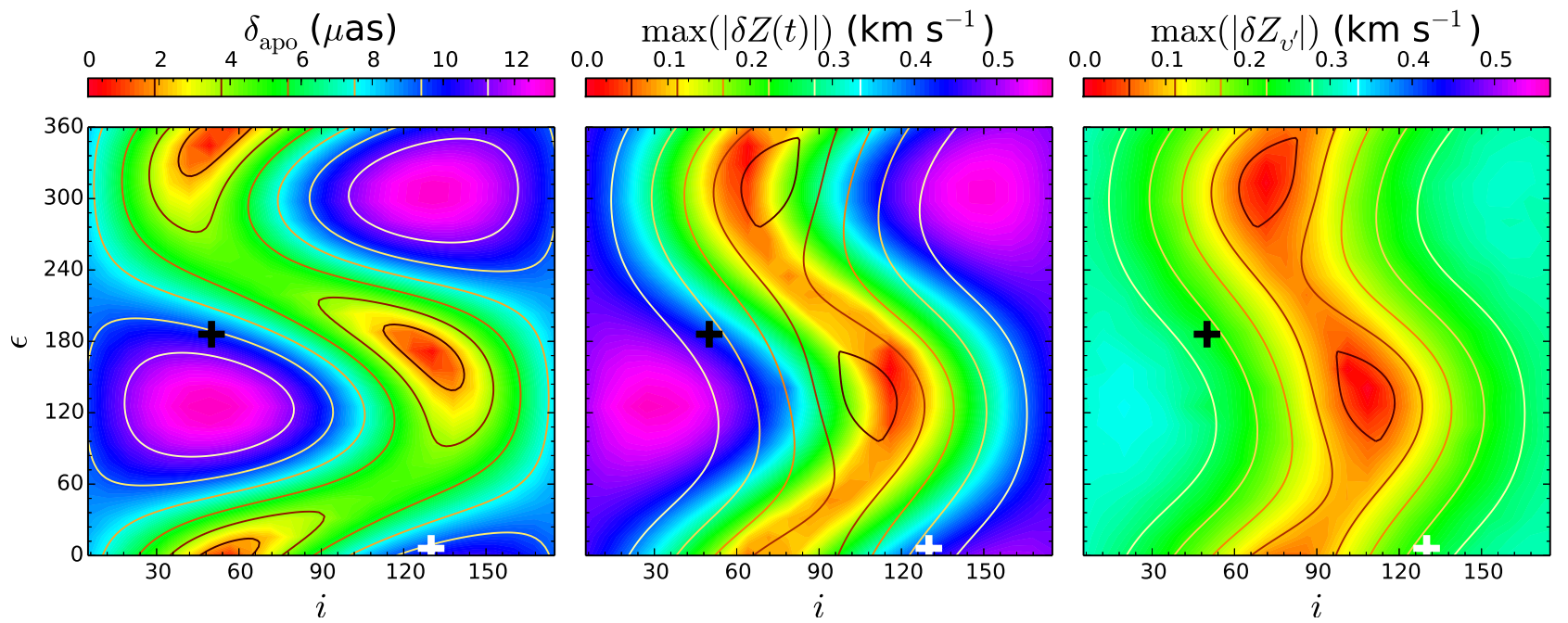

FIG. 2.- Dependence of the spin-induced signals on the spin direction $(i, \epsilon)$ for S2/S0-2 (see Fig. (1) by assuming the MBH spin $a=0.99$. The left and the middle panels show the spin-induced position displacements at apoapsis and the maximum values of the spin-induced redshift differences (defined as $\delta Z(t)$ in Equation 22 after the motion of one full orbit, respectively. The orbital true anomalies $v^{\prime}$ at the same time $t$ are slightly different for different spins and the spin-induced signals caused by the difference in true anomalies are included in the middle panel. For comparison with some analytical results, the right panel shows the maximum redshift differences caused by the spin-induced changes of the orbital plane (defined as $\max \left(\left|\delta Z_{\nu^{\prime}}(t)\right|\right)$, see Eq. 223), with the signals caused by the difference in true anomalies removed. In each panel, the color maps represent the results obtained from the full GR calculations with color indices shown at the top, and the color contour lines represent the analytical ones estimated from Equation (21) in the left panel and Equation (23) in the middle and the right panels. The "+" symbols mark the normal of the orbital plane of the clockwise rotating stellar disk located at the sub-parsec scale of the GC (Yelda et al. 2014). As seen from the figure, the strength of the maximum and the minimum of the spin-induced signals can differ by up to two orders of magnitude, and the spin-induced signals are close to the maximum signals if the spin direction is the same as the normal of the young stellar disk. See Sections 5.1 and 5.2
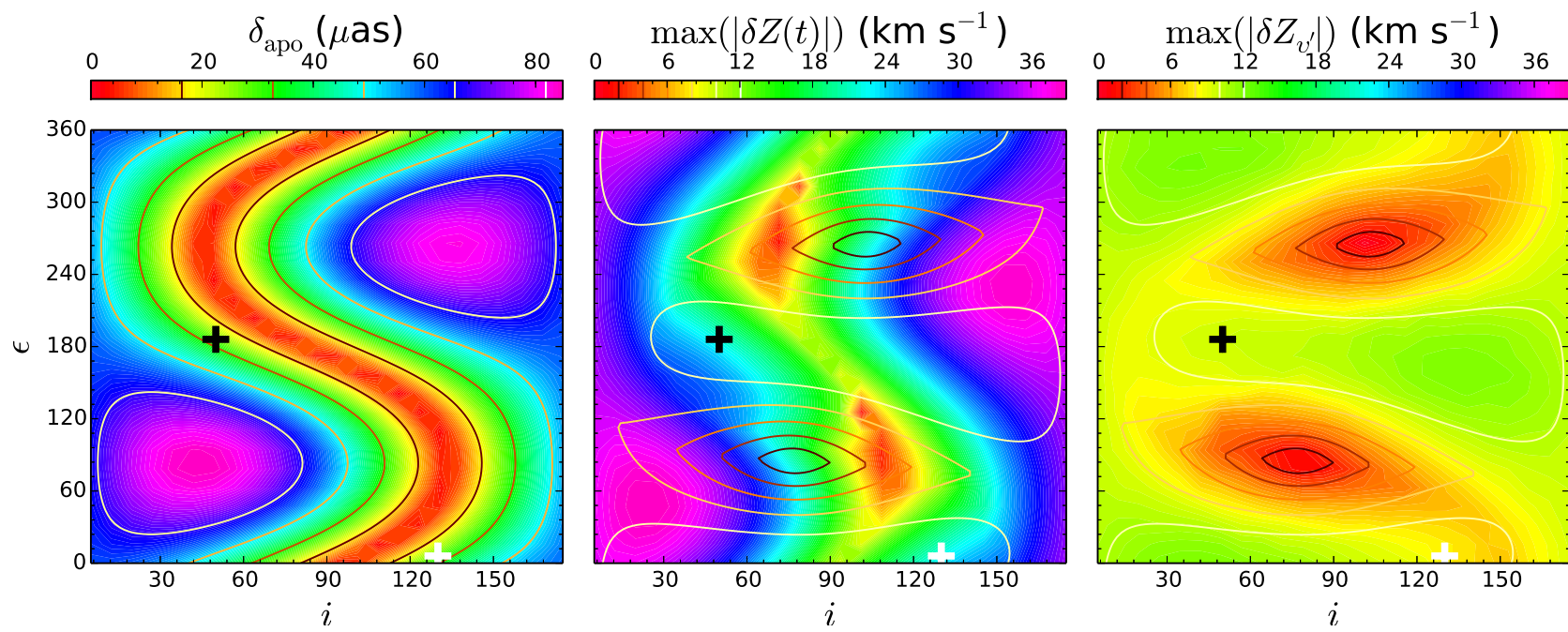

FIG. 3.- Legends similar to Fig. 2 but for a star with a semimajor axis of $a_{\text {orb }}=300 \mathrm{AU}$, an eccentricity of $e_{\mathrm{orb}}=0.95$, and the same orbital orientation as that of S0-102. As seen from the figure, the strength of the spin-induced signals are at the same order of magnitude as the maximum strength if the spin direction is the same as the normal of the young stellar disk. The spin-induced signals are stronger than those shown in Figure 2

and $\Upsilon$ are defined for the star relative to the equatorial plane of the Kerr $\mathrm{BH}$ in the $(x, y, z)$ frame,

$$
\zeta=\arccos \left(\hat{\mathbf{a}} \cdot \hat{\mathbf{n}}_{*}\right)=\arccos \left[\cos I^{\prime} \cos i-\sin I^{\prime} \sin i \sin \left(\Omega^{\prime}-\epsilon\right)\right],
$$

where $\hat{\mathbf{a}}$ is the unit vector along the spin direction, $\hat{\mathbf{n}}_{*}$ is the normal vector of the star's orbital plane, and $a_{\text {orb }}$ is in units of $r_{\mathrm{g}}$. The quadrupole (Q) term of the MBH can also lead to orbital precession by

$$
\delta \Omega_{\mathrm{Q}}=-3 \pi a^{2} \cos \zeta a_{\mathrm{orb}}^{-2}\left(1-e_{\mathrm{orb}}^{2}\right)^{-2}
$$

and

$$
\delta \Upsilon_{\mathrm{Q}}=1.5 \pi a^{2}\left(1-5 \cos ^{2} \zeta\right) a_{\mathrm{orb}}^{-2}\left(1-e_{\mathrm{orb}}^{2}\right)^{-2}
$$

(e.g., Barker \& O'Connell 1975; Wex \& Kopeikin 1999). The changes of $\Omega$ and $\Upsilon$ are then approximately given by

$$
\delta \Omega=\delta \Omega_{\mathrm{LT}}+\delta \Omega_{\mathrm{Q}}
$$

and

$$
\delta \Upsilon=\delta \Upsilon_{\mathrm{LT}}+\delta \Upsilon_{\mathrm{Q}}
$$

Considering the projection effects, the changes of $\Omega^{\prime}, \Upsilon^{\prime}$, 

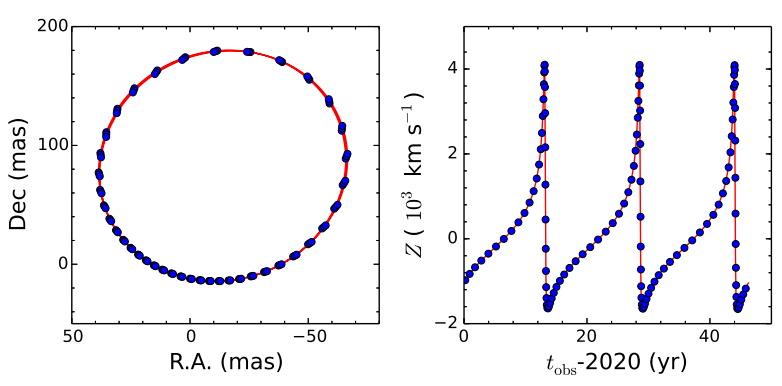

(a)

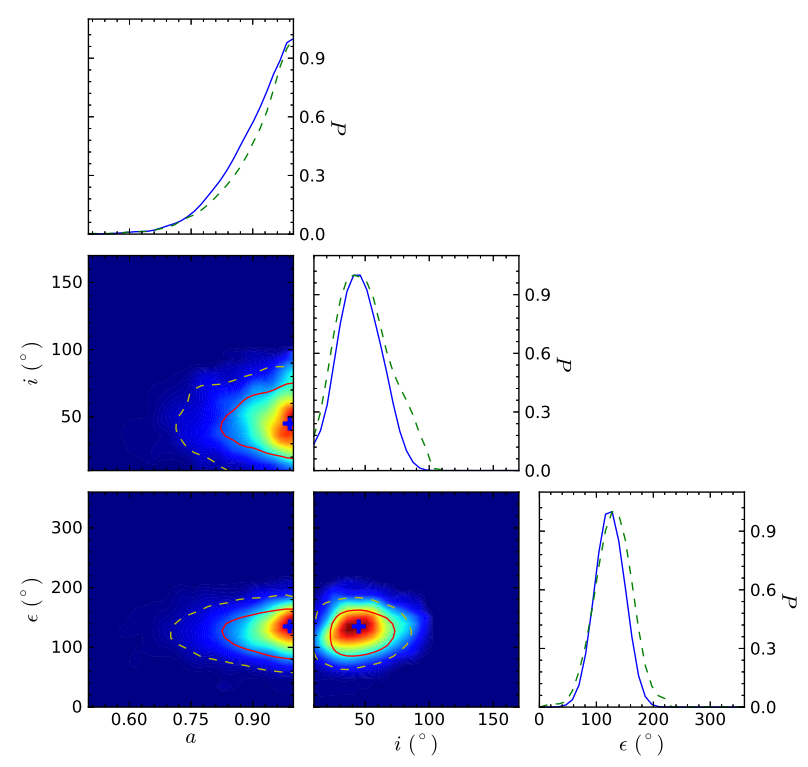

(b)

FIG. 4.- Best fits to the mock observations of S2/S0-2. Panel (a) shows the best fits (red lines) to the mock observations (blue solid circles) on the apparent positions (left) and redshifts (right). Panel (b) shows the 2D contours and the 1D probabilities of parameters $(a, i, \epsilon)$ obtained from the fitting of the mock observations of $\mathrm{S} 2 / \mathrm{S} 0-2$ over three full orbits by assuming intrinsically $(a, i, \epsilon)=\left(0.99,49^{\circ}, 125^{\circ}\right)$. In the $2 \mathrm{D}$ maps, the red solid and the yellow dashed lines show the $1-\sigma$ and the $2-\sigma$ confidence levels, respectively; in the panels for the $1 \mathrm{D}$ probabilities, the blue solid and the green dashed lines show the 1D marginalized distribution and the 1D mean likelihood, respectively. This figure illustrates an example that the spin of the GC MBH and its direction can be constrained well. See details in Section 5.3.1

and $I^{\prime}$ are

$$
\begin{gathered}
\delta \Omega^{\prime}=\left[\cos i+\cos I^{\prime} \sin i \sin \left(\Omega^{\prime}-\epsilon\right) / \sin I^{\prime}\right] \delta \Omega, \\
\delta \Upsilon^{\prime}=-\sin i \sin \left(\Omega^{\prime}-\epsilon\right) / \sin I^{\prime} \delta \Omega+\delta \Upsilon,
\end{gathered}
$$

and

$$
\delta I^{\prime}=-\sin i \cos \left(\Omega^{\prime}-\epsilon\right) \delta \Omega,
$$

respectively.

Position displacement at apoapsis: after the motion of one full orbit, the position displacement at the apoapsis of a star rotating around an $\mathrm{MBH}$ with spin $a$ is approximately

$$
\begin{aligned}
\delta_{\mathrm{apo}} \sim & a_{\mathrm{orb}}\left(1+e_{\mathrm{orb}}\right)\left[\left(1-\sin ^{2} \Upsilon^{\prime} \sin ^{2} I^{\prime}\right) \delta^{2} \Omega^{\prime}\right. \\
& +\left(1-\cos ^{2} \Upsilon^{\prime} \sin ^{2} I^{\prime}\right) \delta^{2} \Upsilon^{\prime} \\
& +2 \cos I^{\prime} \delta \Omega^{\prime} \delta \Upsilon^{\prime}+\sin ^{2} \Upsilon^{\prime} \sin ^{2} I^{\prime} \delta^{2} I^{\prime}
\end{aligned}
$$

$$
\left.-\frac{1}{2} \sin 2 \Upsilon^{\prime} \sin 2 I^{\prime} \delta \Upsilon^{\prime} \delta I^{\prime}-\sin 2 \Upsilon^{\prime} \sin I^{\prime} \delta \Omega^{\prime} \delta I^{\prime}\right]^{1 / 2}
$$

When the quadrupole term is not significant, we have $\delta_{\text {apo }} \propto a_{\text {orb }}^{-1 / 2}\left(1-e_{\text {orb }}^{2}\right)^{-3 / 2}$.

Redshift difference: we consider two cases, one for a star with given initial orbital elements rotating around a non-spinning $\mathrm{MBH}$ with mass $\left(M_{\bullet}\right)$, and the other for a star with the same initial orbital elements but rotating around a rapidly rotating $\mathrm{MBH}$ with the same mass $\left(M_{\bullet}\right)$. The redshift difference between these two cases at a given time $t$ measured by a distant observer is given by

$$
\left.\delta Z(t) \sim \delta Z_{v^{\prime}}\right|_{\mathrm{LT}+\mathrm{Q}}+\delta Z_{\delta v^{\prime}},
$$

where $\left.\delta Z_{v^{\prime}}\right|_{\mathrm{LT}+\mathrm{Q}}$ is the redshift difference at a fixed $v^{\prime}$ due to the spin-induced (LT and Q) precessions and $\delta Z_{\delta v^{\prime}} \equiv \frac{\partial Z_{v^{\prime}}}{\partial v^{\prime}} \delta v^{\prime}$ is the redshift difference due to the slight difference between the true anomalies $\left(v^{\prime}\right)$ of these two stars at a given time $t$ caused by the difference of the spin values. The two terms in Equation (22) can be roughly given by the projection of the spin-induced Keplerian velocity difference at the line of sight, i.e.,

$$
\begin{aligned}
\left.\delta Z_{v^{\prime}}\right|_{\mathrm{LT}+\mathrm{Q}} \sim & -\left[\frac{G M_{\bullet}}{a_{\mathrm{orb}}\left(1-e_{\mathrm{orb}}^{2}\right)}\right]^{1 / 2}\left\{\operatorname { c o s } I ^ { \prime } \left[e_{\mathrm{orb}} \cos \Upsilon^{\prime}\right.\right. \\
& \left.+\cos \left(\Upsilon^{\prime}+v^{\prime}\right)\right] \delta I^{\prime}-\sin I^{\prime}\left[e_{\mathrm{orb}} \sin \Upsilon^{\prime}\right. \\
& \left.\left.+\sin \left(\Upsilon^{\prime}+v^{\prime}\right)\right] \delta \Upsilon^{\prime}\right\}, \\
\delta Z_{\delta v^{\prime}} \sim- & {\left[\frac{G M_{\bullet}}{a_{\mathrm{orb}}\left(1-e_{\mathrm{orb}}^{2}\right)}\right]^{1 / 2} \sin I^{\prime} \sin \left(\Upsilon^{\prime}+v^{\prime}\right) \delta v^{\prime}(t) . }
\end{aligned}
$$

The maximum difference of $\left.\delta Z_{v^{\prime}}\right|_{\mathrm{LT}+\mathrm{Q}}$ over a full orbit can be obtained from Equation (23) analytically (see Zhang et al. 2015). However, $\delta v^{\prime}$ may only be obtained numerically for a star with arbitrary orbital elements. For the following comparisons between the numerical and the analytical results of the spin-induced redshift differences, we therefore mainly focus on $\left.\delta Z_{v^{\prime}}\right|_{\mathrm{LT}+\mathrm{Q}}$. Note that $\left.\delta Z_{v^{\prime}}\right|_{\mathrm{LT}+\mathrm{Q}} \propto a_{\mathrm{orb}}^{-2}\left(1-e_{\mathrm{orb}}^{2}\right)^{-2}$ if $e_{\mathrm{orb}} \rightarrow 1$, and $\left.\delta Z_{v^{\prime}}\right|_{\mathrm{LT}+\mathrm{Q}} \propto a_{\mathrm{Orb}}^{-2}$ if $e_{\mathrm{orb}} \rightarrow 0$, which are consistent with the findings by Angélil \& Saha (2010).

In the derivation of Equations (21) and (23), we omit the effects due to photon propagation from the star to the distant observer. For those photons emitted from the star at different locations, their trajectories may be bended and their energy may be shifted differently.

\subsubsection{Comparison}

As shown in the left panels of Figures 2 and 3 , the position displacements at the apoapsis obtained from Equation (21) for S2/S0-2 and the hypothetical star are quite consistent with the numerical ones. The residuals between the full GR results and the analytical ones are on the order of one percent (for $\mathrm{S} 2 / \mathrm{S} 0-2$ ) to ten percent (for the hypothetical star) of the full GR results, which are mainly caused by the omission of the photon propagation effects and the high-order precessions.

The middle panels of Figures 2 and 3 show both the full GR results on the spin-induced maximum redshift differences over one full orbit and the analytical ones due to the LT and Q precessions (Eq. 23) for S2/S0-2 

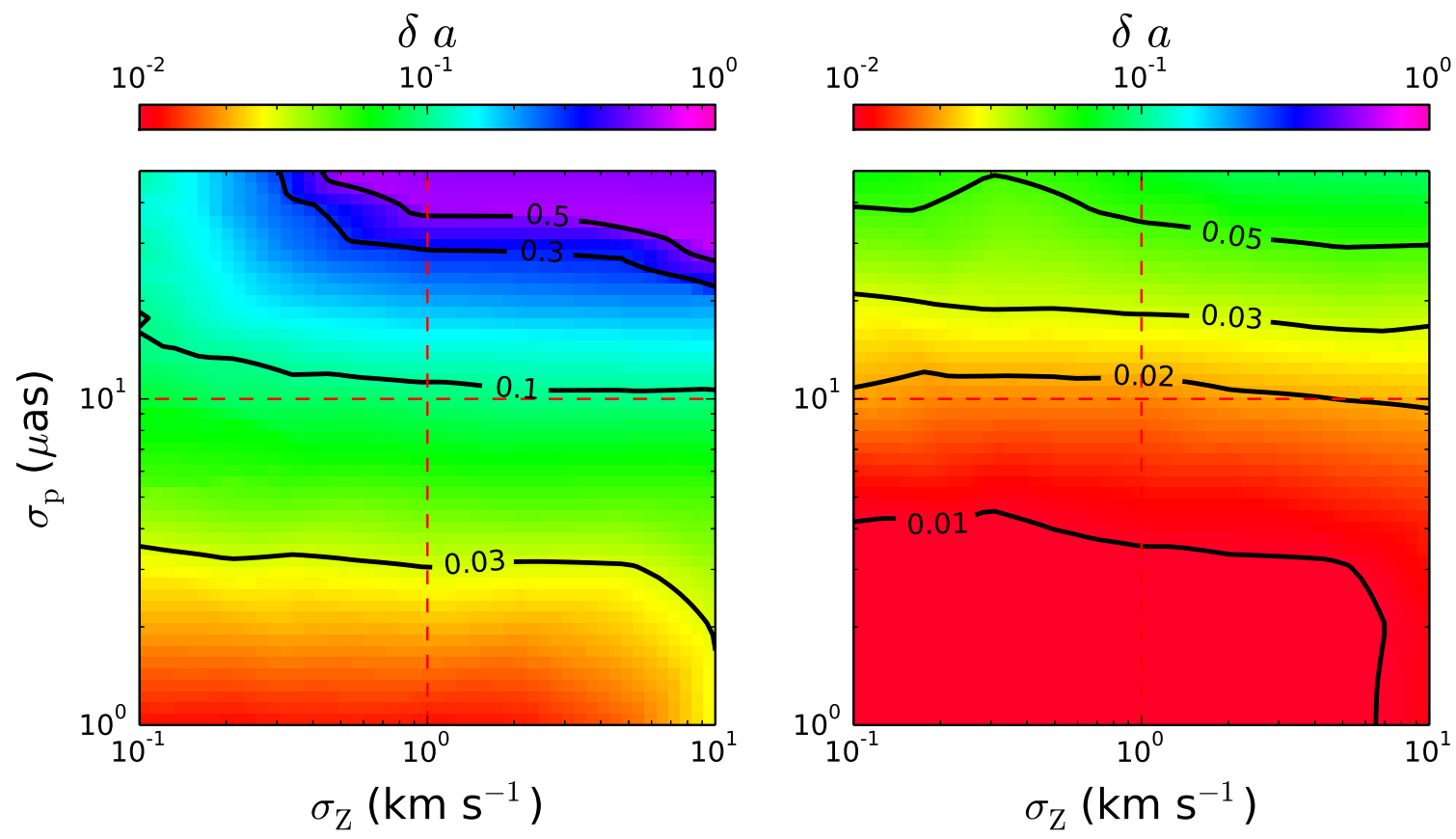

FIG. 5.- Dependence of the quality of the MBH spin constraints $(\delta a)$ on the astrometric precision $\left(\sigma_{\mathrm{p}}\right)$ and the velocity precision $\left(\sigma_{z}\right)$, which are obtained from mock observations of two different stars over three full orbits. The left panel shows the result obtained from the mock observations of S2/S0-2 by assuming $(a, i, \epsilon)=\left(0.99,49^{\circ}, 125^{\circ}\right)$; and the right panel shows the result obtained from the mock observations of a hypothetical star with $\left(a_{\mathrm{orb}}, e_{\mathrm{orb}}\right)=(300 \mathrm{AU}, 0.95)$ and an orientation that is the same as that of S0-102, by assuming $(a, i, \epsilon)=\left(0.99,49^{\circ}, 125^{\circ}\right)$. The top color bars indicate the value of $\delta a$. The black lines represent the contours of $\delta a$ with the marked values. The red dashed lines in both panels mark the astrometric and the velocity precisions to be possibly achieved by the next-generation facilities. As seen from the figure, in the ranges of astrometric precision and velocity precision with $1-30 \mu a s$ and $0.1-10 \mathrm{~km} \mathrm{~s}^{-1}$, an improvement in astrometric precision would be more effective at improving the quality of constraining the spin than an improvement in velocity precision.

and the hypothetical star, respectively. Apparently, the numerical results deviate significantly from the analytical ones derived from Equation (23), mainly because of the omission of the second term in Equation (22) in the analytical estimates. The right panels of Figures 2 and 3 show the full GR results on the spin-induced redshift difference obtained by numerically removing the part $\delta Z_{\delta v^{\prime}}$ for $\mathrm{S} 2 / \mathrm{S} 0-2$ and the hypothetical star, respectively. As seen from these two panels, the full GR results are quite consistent with the analytical ones. However, our calculations show that the residuals between the full GR results and the analytical results are on the order of a few to a few tens percent for both $\mathrm{S} 2 / \mathrm{S} 0-2$ and the hypothetical star, mainly due to the omission of the photon propagation effects and the high-order precessions. Our calculations show that the spin-induced effects on the photon propagation and the rotation velocity at a given position can introduce some differences in the redshifts, though about 5-10 times smaller than that introduced by the position shift (Eq. 23) for S2/S0-2 and the hypothetical star. Therefore, the photon propagation effects cannot be neglected when considering an accurate constraint on the MBH spin and metric.

The contribution of the $\mathrm{Q}$ precession (e.g., $\delta \Omega_{\mathrm{Q}}$ and $\left.\delta \Upsilon_{\mathrm{Q}}\right)$ to the spin-induced effects is only about one to a few percent, substantially smaller than that contributed by the LT precession, for stars with semimajor axes $\sim 300-1000 \mathrm{AU}$ and eccentricities $\gtrsim 0.88$. Our calculations show that the deviations of the analytical results on the spin-induced position displacements and redshift differences from those numerical ones are on percentage or even a higher level, comparable to the contribution from the quadrupole moment, mainly because of the omission of the photon propagation effects and the high-order precessions in obtaining the analytical results (see details in Zhang et al. 2015 and Angélil et al. 2010). Therefore, it is important to use the full GR numerical method to account for the photon propagation effects when considering constraints on the $\mathrm{MBH}$ spin with high precision (e.g., percentage level) or the quadrupole moment.

\subsection{Orbital reconstruction and the quality of constraining the $\mathrm{MBH}$ spin}

Using the full GR method introduced above, we can generate mock observations on both the trajectory of the apparent position and the redshift curve measured by a distant observer for any star, e.g., S2/S0-2 or S0-102, with any given set of astrometric and velocity precisions $\left(\sigma_{\mathrm{p}}, \sigma_{Z}\right)$; and then we use the mock observation results to reconstruct orbital elements of the star and the intrinsic properties of the $\mathrm{MBH}$ by the MCMC fitting technique as follows. The trajectory and the redshift curve of a star are determined by 11 parameters $(\boldsymbol{\Theta})$, including the $\mathrm{MBH}$ properties $\left(M_{\bullet}, a, i, \epsilon\right)$, the distance from the $\mathrm{MBH}$ to the sun $\left(R_{\mathrm{GC}}\right)$, and the star's initial orbital elements $\left(a_{\text {orb }, 0}, e_{\mathrm{orb}, 0}, \Omega_{0}^{\prime}, I_{0}^{\prime}, \Upsilon_{0}^{\prime}, v_{0}^{\prime}\right)$ 叫

4 Note that here we ignore the possible velocity of the $\mathrm{MBH}$ relative to $\mathrm{Sgr} \mathrm{A}^{*}$ and the acceleration of the $\mathrm{MBH}$ with respect to the observer. In principle, both the velocity and the acceleration can be added to the fitting as free parameters and constrained by the observations. By doing so, the motion of the solar system may also be constrained simultaneously with a high accuracy, since the pattern of the relative motion of the observer to the GC $\mathrm{MBH}$ is different from the spin-induced motion. See a detailed discussion in Zhang et al. (2015). 
To obtain mock observations, we set $M_{\bullet}=4 \times$ $10^{6} M_{\odot}, R_{\mathrm{GC}}=8 \mathrm{kpc}$. For a given set of mock observations on both apparent position and redshift $\mathbf{D}=$ $\left(\alpha_{\mathrm{obs}, k}, \beta_{\mathrm{obs}, k}, Z_{\mathrm{obs}, k}\right)$ at time $t_{\mathrm{obs}, k}(k=1,2, \ldots, N$ and $N$ is the total number of the mock observations), we can use the full GR method to generate model results on $\left(\alpha_{k}, \beta_{k}, Z_{k}\right)$ at $t_{\mathrm{obs}, k}$ for any given set of parameters $\boldsymbol{\Theta}$. We then adopt the MCMC technique to fit the mock observations and obtain the best fit of each parameter, i.e., the posteri probability of the model parameters

$$
P(\boldsymbol{\Theta} \mid \mathbf{D}) \propto \exp \left(-\chi^{2} / 2\right) P(\boldsymbol{\Theta}) .
$$

Here

$$
\begin{aligned}
\chi^{2}= & \sum_{k_{1}=1}^{N_{1}} \frac{\left(\alpha_{k_{1}}-\alpha_{\mathrm{obs}, k_{1}}\right)^{2}+\left(\beta_{k_{1}}-\beta_{\mathrm{obs}, k_{1}}\right)^{2}}{\sigma_{\mathrm{p}}^{2}} \\
& +\sum_{k_{2}=1}^{N_{2}} \frac{\left(Z_{k_{2}}-Z_{\mathrm{obs}, k_{2}}\right)^{2}}{\sigma_{Z}^{2}}
\end{aligned}
$$

and $P(\boldsymbol{\Theta})$ is the prior probability distribution and assumed to be a flat distribution, $N_{1}$ and $N_{2}$ are the number of mock observations for the position and the redshift of the target star, respectively.

\subsubsection{Examples}

We first generate mock observations for $\mathrm{S} 2 / \mathrm{S} 0-2$ over three full orbits by assuming $(a, i, \epsilon)=\left(0.99,49^{\circ}, 125^{\circ}\right)$ and $\left(\sigma_{\mathrm{p}}, \sigma_{Z}\right)=\left(10 \mu \mathrm{as}, 1 \mathrm{~km} \mathrm{~s}^{-1}\right)$. With this assumed spin direction, the spin-induced position displacement for $\mathrm{S} 2 / \mathrm{S} 0-2$ are the most significant. The total number of mock observations are set to $N_{1}=N_{2}=120$. We take more mock observations near the periapsis than that near the apoapsis. Figure 4 shows both the fittings to the mock observations (panel (a)) and the two-dimensional (2D) probability contours and onedimensional (1D) marginalized probability distributions for the spin parameters $(a, i, \epsilon)$ (panel (b)). According to the fitting, we find that the spin value $a$ is constrained to $0.90_{-0.15}^{+0.09}$, well consistent with the input one; and the spin direction can be simultaneously constrained with $(i, \epsilon)=\left(46^{\circ}+26^{\circ}, 124^{\circ}+38^{\circ},{ }^{\circ}\right)$. In the mean time, the $\mathrm{MBH}$ mass and the distance from the $\mathrm{MBH}$ to the sun can be constrained to a relative accuracy of $\sim 1-2 \times 10^{-4}$.

The spin-induced effects are moderate (see Fig. 2) if the spin direction is close to the orientation of the clockwise rotating stellar disk $(i, \epsilon)=\left(130^{\circ}, 6^{\circ}\right)$ or $\left(50^{\circ}, 186^{\circ}\right)$ in the GC (Yelda et al. 2014). For this case, the spin-induced effects on both the position and the redshift are moderate so that the spin and its direction can still be well constrained

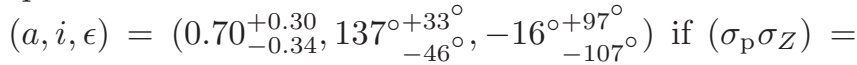
(10 $\left.\mu \mathrm{as}, 1 \mathrm{~km} \mathrm{~s}^{-1}\right)$.

If the spin direction is $(i, \epsilon)=\left(50^{\circ}, 351^{\circ}\right)$ or $\left(130^{\circ}, 171^{\circ}\right)$, the spin-induced effects on the position displacements are the least and those on the redshift differences are also close to the least, and thus the spin and its direction cannot be well constrained even if $\left(\sigma_{\mathrm{p}}, \sigma_{Z}\right)=\left(10 \mu \mathrm{as}, 1 \mathrm{~km} \mathrm{~s}^{-1}\right)$.

By surveying the parameter space, we find that the spin can be well constrained for about half of the possi- ble spin directions shown in Figure 2 by using the motion of three full orbits of S2/S0-2 if $a$ is close to 1 and $\left(\sigma_{\mathrm{p}}, \sigma_{Z}\right) \sim\left(10 \mu \mathrm{as}, 1 \mathrm{~km} \mathrm{~s}^{-1}\right)$. With such expected position and redshift precisions, the $\mathrm{GC} \mathrm{MBH}$ spin may be better constrained via the motion of $\mathrm{S} 2 / \mathrm{S} 0-2$ if choosing a substantially longer monitoring period (but impractical); however, a robust constraint on the spin would be difficult to obtain via the motion of $\mathrm{S} 2 / \mathrm{S} 0-2$ if choosing a substantially shorter monitoring period (e.g., a decade) and considering that the spin is likely not to point just at the direction with the most significant spin-induced effects.

\subsubsection{Dependence on $\left(\sigma_{\mathrm{p}}, \sigma_{Z}\right)$}

We further generate mock observations for $\mathrm{S} 2 / \mathrm{S} 0-2$ over three full orbits by assuming various combinations of $\sigma_{\mathrm{p}}$ and $\sigma_{Z}$, and then we adopt the MCMC technique to obtain the constraint on the $\mathrm{MBH}$ spin for each set of $\left(\sigma_{\mathrm{p}}, \sigma_{Z}\right)$ as $a_{-\delta a_{2}}^{+\delta a_{1}}$, where $\delta a_{1}$ and $\delta a_{2}$ are the $1-\sigma$ errors obtained from each fit. We set $\delta a=\max \left(\left|\delta a_{1}\right|,\left|\delta a_{2}\right|\right)$ to represent the quality of the spin constraint. The left panel of Figure 5 shows the color map of $\delta a$ on the $\sigma_{\mathrm{p}}-\sigma_{Z}$ plane. For an $\mathrm{MBH}$ with $(a, i, \epsilon)=\left(0.99,49^{\circ}, 125^{\circ}\right)$, as seen from this panel, the $\mathrm{MBH}$ spin can be constrained to an accuracy of $\delta a \sim 0.1$ over a period of $\sim 45 \mathrm{yr}$ if $\left(\sigma_{\mathrm{p}}, \sigma_{Z}\right)=\left(10 \mu \mathrm{as}, 1 \mathrm{kms}^{-1}\right)$. If the position and the redshift precisions can be improved by a factor of a few, then the GC MBH spin may be constrained with an even higher accuracy within the same time period or within a relatively shorter time period (e.g., a decade) with the same accuracy.

We also generate mock observations for the hypothetical star over three full orbits and use the MCMC technique to obtain the constraint on the $\mathrm{MBH}$ spin with the mock observations. This star has a semimajor axis of $300 \mathrm{AU}$, an eccentricity of 0.95 and an orbital orientation the same as that of S0-102. The right panel of Figure 5 shows the dependence of the quality of the spin constraint $\delta a$ on the position and the redshift precisions obtained from the mock observations of this star. For an $\mathrm{MBH}$ with $(a, i, \epsilon)=\left(0.99,49^{\circ}, 125^{\circ}\right)$, as seen from this panel, the MBH spin can be constrained to an accuracy of $\delta a \lesssim 0.02$ over a period of $\sim 10 \mathrm{yr}$ if $\left(\sigma_{\mathrm{p}}, \sigma_{Z}\right)=\left(10 \mu \mathrm{as}, 1 \mathrm{~km} \mathrm{~s}^{-1}\right)$. Since the position and the redshift differences induced by the precession due to the quadrupole moment are about one percent to ten percent of the total spin-induced signals, this star, if existing, can be used to put a constraint on the quadrupole moment and thus possibly test the 'no-hair' theorem. Note that Will (2008) argued that at least two stars are needed to disentangle the spin and the quadrupole moment, as the difference in the orbital configurations of the two stars can help to break some degeneracy and determine the involved parameters. Here even a single star can do this, because the orbital configuration of the star evolves with time and the evolution effects are incorporated into the time series of the mock observations, given the sufficiently high measurement precisions and a long observational period. Similarly, it was proposed that the spin and the quadrupole moment of the central $\mathrm{MBH}$ can also be measured simultaneously by one pulsar given a high timing precision (Liu et al. 2012).

Although Figure 5 is obtained by setting the spin 

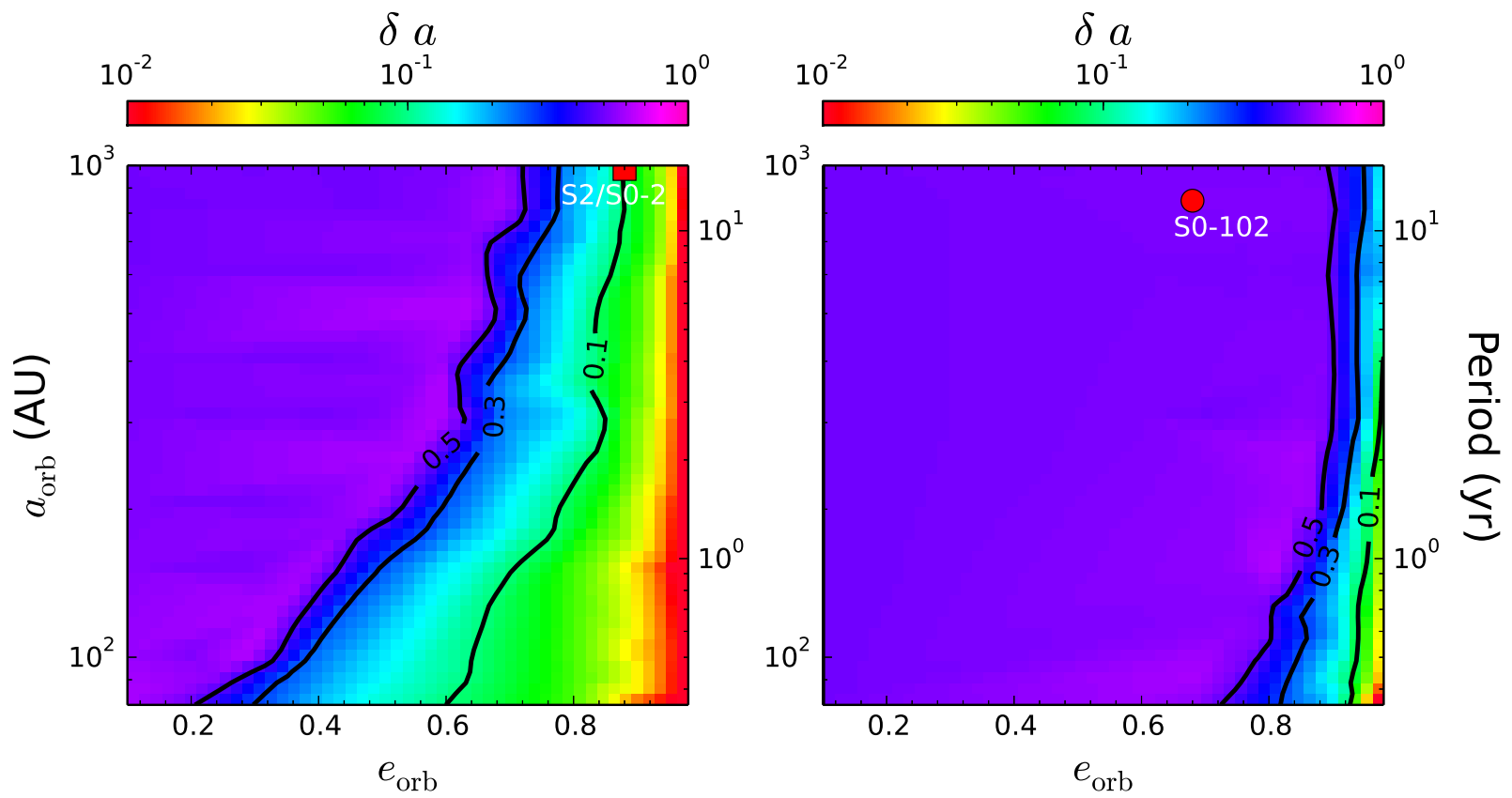

FIG. 6. - Dependence of the quality of the spin constraints $(\delta a)$ on the semimajor axis (or the orbital period) and the eccentricity of hypothetical stars. The left panel shows the simulation results obtained for stars with orbital orientations that are the same as that of S2/S02 and assuming $(a, i, \epsilon)=\left(0.99,49^{\circ}, 125^{\circ}\right),\left(\sigma_{\mathrm{p}}, \sigma_{z}\right)=\left(10 \mu \mathrm{as}, 1 \mathrm{~km} \mathrm{~s}^{-1}\right)$; and the right panel shows the simulation results obtained for those stars with orbital orientations that are the same as that of S0-102 and assuming $(a, i, \epsilon)=\left(0.99,49^{\circ}, 125^{\circ}\right),\left(\sigma_{\mathrm{p}}, \sigma_{z}\right)=\left(50 \mu \mathrm{as}, 5 \mathrm{~km} \mathrm{~s}{ }^{-1}\right)$. The top color bar indicates the value of $\delta a$. The black lines represent the contours of $\delta a$ with the marked values. The solid square in the left panel and the solid circle in the right panel mark the positions of S2/S0-2 and S0-102, respectively (Mever et al. 2012). In the figure, the spin of the GC MBH can be constrained with $1-\sigma$ error $\lesssim 0.1$ (right panel) or even $\lesssim 0.02$ (left panel) by monitoring the orbital motion of a star with semimajor axis $\lesssim 300 \mathrm{AU}$ and eccentricity $\gtrsim 0.95$ over a period of $\lesssim 10 \mathrm{yr}$.

$a=0.99$, it can be applied to other spin values if the spin has the same direction as that in Figure 5. (For the region with $\delta a \gtrsim a$, the $\mathrm{MBH}$ spin cannot be constrained distinctively from the case with zero spin.) If the spin direction is different from the one with the maximum spin-induced effects, the spin-induced position displacement and redshift difference may be a factor of $D$ and $F$ $(D, F>1)$ smaller than the maximum ones, where the factors $D$ and $F$ can be derived from Figures 2 and 3 . Therefore, the map for the quality of the spin constraint can also be roughly obtained by replacing the figure labels $\left(\sigma_{\mathrm{p}}\right.$, and $\left.\sigma_{Z}\right)$ in both panels of Figure 5 by $D \sigma_{\mathrm{p}}$ and $F \sigma_{Z}$, respectively. We have checked this numerically and find that it is appropriate.

\subsubsection{Dependence on $\left(a_{\mathrm{orb}}, e_{\mathrm{orb}}\right)$}

The spin-induced signals depend on the semimajor axis and the eccentricity of the star. The left panel of Figure 6 shows the quality of the spin constraints by using the motion of three full orbits of hypothetical stars with the same orbital orientation as that of $\mathrm{S} 2 / \mathrm{S} 0-2$ but with various semimajor axes and eccentricities. The input spin and its direction are $(a, i, \epsilon)=\left(0.99,49^{\circ}, 125^{\circ}\right)$ and the position and the redshift precisions are $\left(\sigma_{\mathrm{p}}, \sigma_{Z}\right)=$ $\left(10 \mu \mathrm{as}, 1 \mathrm{~km} \mathrm{~s}^{-1}\right)$. Three orbital periods of a star with $a_{\text {orb }} \simeq 300$ AU are about 10 yr. As shown in the figure, the spin and its direction can be accurately constrained $(\delta a \lesssim 0.02)$ within a short period (e.g., a decade) by using the motion of a star with $a_{\text {orb }} \lesssim 300 \mathrm{AU}$ and $e_{\text {orb }} \gtrsim 0.95$. In this case, a constraint on the quadrupole moment is also possible since the contribution from the quadrupole moment can be as high as a few to ten per- cent of the total spin-induced position displacements and redshift differences. With such a star and the assumed position and redshift precisions $\left(10 \mu \mathrm{as}, 1 \mathrm{~km} \mathrm{~s}^{-1}\right)$, the $\mathrm{MBH}$ spin can still be well constrained if the spin is not pointing close to the direction with the least spin-induced effects, according to the difference in the signals with different spin directions as shown in Figure 3 . However, a star with $a_{\text {orb }} \gtrsim 300 \mathrm{AU}$ and $e_{\text {orb }} \lesssim 0.6$ may be not so useful in constraining the GC MBH spin within a relatively short period (e.g., a decade), according to the left panel of Figure 6.

The right panel of Figure 6 shows the quality of the spin constraints by using the motion of three full orbits of hypothetical stars with the same orbital orientation as that of S0-102 and assuming the position and the redshift precisions of $\left(\sigma_{\mathrm{p}}, \sigma_{Z}\right)=\left(50 \mu \mathrm{as}, 5 \mathrm{~km} \mathrm{~s}^{-1}\right)$. The input spin and its direction are $(a, i, \epsilon)=\left(0.99,49^{\circ}, 125^{\circ}\right)$. For such a spin direction, the spin-induced effects are moderate, though not maximum, as illustrated in Figure 3 . As shown in the right panel of Figure 6. the spin can be constrained to an accuracy of $\delta a \lesssim 0.1-0.3$ by using the motion over a few orbits of a star with $a_{\text {orb }} \lesssim 300 \mathrm{AU}$ and $e_{\text {orb }} \gtrsim 0.95$ even if the astrometric and the velocity precisions are only about ( $\left.50 \mu \mathrm{as}, 5 \mathrm{~km} \mathrm{~s}^{-1}\right)$. Furthermore, for more than half of the possible spin directions, the $\mathrm{MBH}$ spin can still be constrained by monitoring the motion of a few orbits of such a star with the assumed position and redshift precisions of $\left(50 \mu \mathrm{as}, 5 \mathrm{~km} \mathrm{~s}^{-1}\right)$.

The spin-induced effects also depend on the orbital orientation of a star rotating around the $\mathrm{MBH}$, and this dependence is partly incorporated into the analytic approximations described by Equations (21) and (23)-(24). 

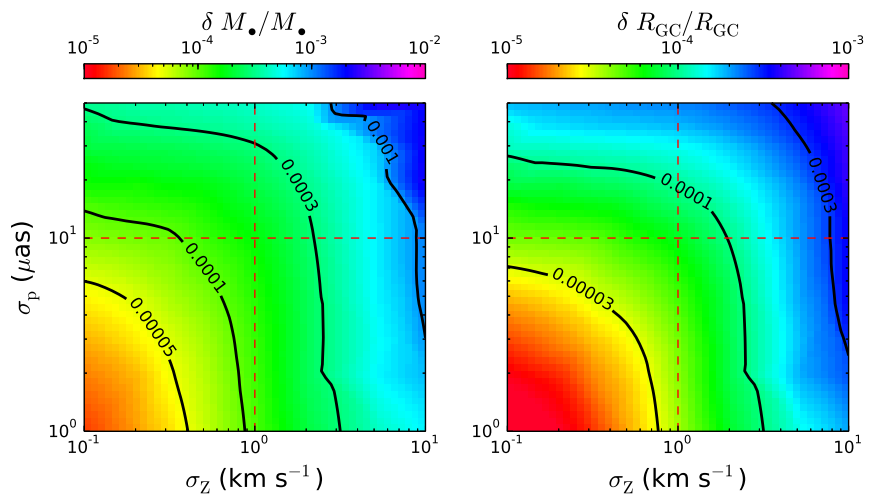

(a)
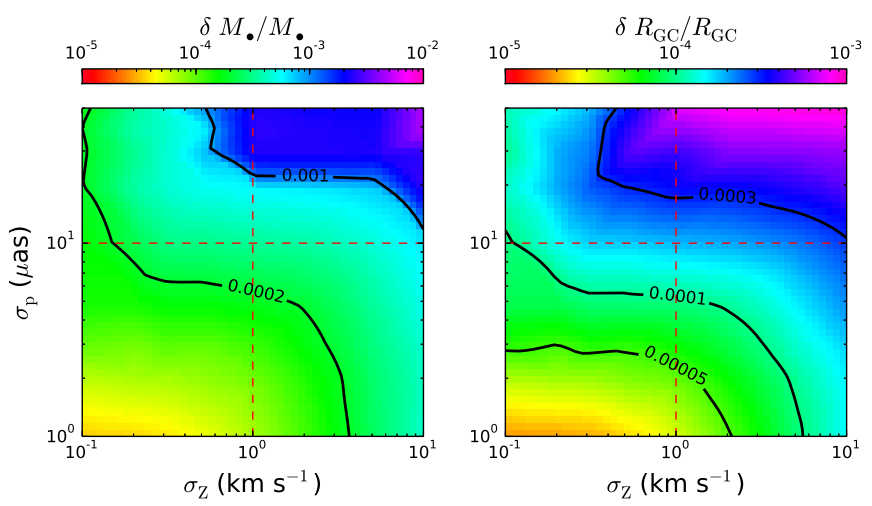

(b)

FIG. 7.- Dependence of the qualities of the MBH mass constraint $\left(\delta M_{\bullet} / M_{\bullet}\right)$ and the $\mathrm{GC}$ distance constraint $\left(\delta R_{\mathrm{GC}} / R_{\mathrm{GC}}\right)$ on the astrometric precision $\left(\sigma_{\mathrm{p}}\right)$ and the velocity precision $\left(\sigma_{Z}\right)$ The top panels show the results obtained from the mock observations of S0-2/S2 by assuming $(a, i, \epsilon)=\left(0.99,49^{\circ}, 125^{\circ}\right)$; and the bottom panels show the results obtained from the mock observations for a hypothetical star with an orientation that is the same as that of S0-102, i.e., $(a, i, \epsilon)=\left(0.99,49^{\circ}, 125^{\circ}\right)$. The color bars indicate the values of $\delta M_{\bullet} / M_{\bullet}$ or $\delta R_{\mathrm{GC}} / R_{\mathrm{GC}}$, as labeled. The black lines represent the contours of $\delta M_{\bullet} / M_{\bullet}$ or $\delta R_{\mathrm{GC}} / R_{\mathrm{GC}}$ with the marked values. The figure indicates that the smaller semimajor axis and the higher eccentricity of a star does not mean a higher-quality constraint on the MBH mass and the GC distance. In addition, although the improvement in velocity precision in the displayed range is not effective at improving the quality of constraining the MBH spin as shown in Figure 5 it can be effective at improving the quality of constraining the $\mathrm{MBH}$ mass and the GC distance. See Section 5.3.4

By applying those analytical approximations to the motion over a few orbits of an assumed star with semimajor axis $\lesssim 300 \mathrm{AU}$, eccentricity $\gtrsim 0.95$, but arbitrary orbital orientations, we find that the $\mathrm{GC} \mathrm{MBH}$ spin can be well constrained for most of the possible spin directions if $\left(\sigma_{\mathrm{p}}, \sigma_{Z}\right)=\left(10 \mu \mathrm{as}, 1 \mathrm{~km} \mathrm{~s}^{-1}\right)$.

\subsubsection{Constraints on the $M B H$ mass and the $G C$ distance}

The qualities of the constraints on the $\mathrm{MBH}$ mass $\left(M_{\bullet}\right)$ and the GC distance $\left(R_{\mathrm{GC}}\right)$ may be defined as $\delta M_{\bullet} / M_{\bullet}$ and $\delta R_{\mathrm{GC}} / R_{\mathrm{GC}}$, respectively, where $\delta M_{\bullet}$ and $\delta R_{\mathrm{GC}}$ are the $1 \sigma$ errors obtained from the MCMC fittings to the mock observations. Figure 7 shows the qualities of the $\mathrm{MBH}$ mass constraint (left panels) and the $\mathrm{GC}$ distance constraint (right panels), respectively, obtained from the mock observations for S0-2/S2 (top panels) and a hypothetical star (bottom panels) for a large range of the position and redshift accuracies. As shown in this figure, the accuracies of the $\mathrm{MBH}$ mass and the $\mathrm{GC}$ dis- tance constraints can be up to a few $10^{-4}$ and $10^{-4}$, respectively, by using the motion of $\mathrm{S} 0-2 / \mathrm{S} 2$ over a few orbits if the astrometric and the velocity accuracy of future facilities can reach $\left(\sigma_{\mathrm{p}}, \sigma_{Z}\right)=\left(10 \mu \mathrm{as}, 1 \mathrm{~km} \mathrm{~s}^{-1}\right)$ as expected. Using a star with a significantly smaller semimajor axis compared to $\mathrm{S} 0-2 / \mathrm{S} 2$, e.g., $a_{\text {orb }}=300 \mathrm{AU}$, the accuracies of the $\mathrm{MBH}$ mass and the $\mathrm{GC}$ distance constraints are poorer than those obtained by using S0$2 / \mathrm{S} 2$ with the same $\sigma_{\mathrm{p}}$ and $\sigma_{Z}$. The main reason is that the relative position error is higher for a star with a smaller semimajor axis given the same astrometric precision. Even if the astrometric precision and the velocity precision are $\left(\sigma_{\mathrm{p}}, \sigma_{Z}\right)=\left(50 \mu \mathrm{as}, 5 \mathrm{~km} \mathrm{~s}^{-1}\right)$, much poorer than the optimistic expectations for the future facilities, the accuracies of the $\mathrm{MBH}$ mass and the GC distance constraints can still be up to one thousandth and a few $10^{-4}$, respectively, by using the motion of S0-2/S2 over three full orbits, although the $\mathrm{MBH}$ spin cannot be well constrained for this case.

\section{SUMMARY AND DISCUSSIONS}

In this paper, we adopt a full GR numerical method to investigate possible constraints on the $\mathrm{GC} \mathrm{MBH}$ spin that may be obtained by future facilities via the relativistic motion of a star surrounding the $\mathrm{MBH}$. In the mapping of the dependence of the spin-induced signals on any spin direction of the $\mathrm{MBH}$ for the given example stars, the maximum and the minimum strength of the spin-induced signals can differ by up to two orders of magnitude, and the strength of the spin-induced signals are at the same order of magnitude as the maximum strength if the spin direction is the same as the normal of the clockwise rotating young stellar disk located at the sub-parsec scale of the GC. The motion of the stars is helpful to test whether those two directions are the same or significantly different and further provide insights into the assembly history of the MBH. We use the MCMC fittings to demonstrate the quality of constraining the $\mathrm{MBH}$ spin, given any set of the astrometric and the redshift precisions of observational facilities. Future facilities, such as the GRAVITY on the VLTI, the TMT, and the E-ELT, are expected to realize position and redshift (or velocity) precisions at the level of $10-50 \mu$ as and $1 \mathrm{kms}^{-1}$, respectively. We find that in the ranges of astrometric precision and velocity precision with 1-30 $\mu$ as and $0.1-10 \mathrm{kms}^{-1}$, an improvement in astrometric precision would be more effective at improving the quality of constraining the spin than an improvement in velocity precision, although the improvement in velocity precision can be effective at improving the quality of constraining the $\mathrm{MBH}$ mass and the GC distance. We obtain the parameter space of the semimajor axis and the eccentricity for the orbit of the target star that a high-precision constraint on the GC MBH spin can be obtained via the motion of the star. We demonstrate that the $\mathrm{GC} \mathrm{MBH}$ spin can be constrained with high precision of $1-\sigma$ error $\lesssim 0.1$ or even $\lesssim 0.02$ by monitoring the motion of a star, if existing, with a semimajor axis of $\lesssim 300 \mathrm{AU}$ and an eccentricity of $\gtrsim 0.95$ in a period of $\lesssim 10 \mathrm{yr}$ with future facilities. With such a star and the optimistically expected position and redshift precisions, the quadrupole moment of the $\mathrm{MBH}$ can also be constrained and thus the 'no-hair' theorem may be tested for optimistic cases in 
which the spin is pointing close to the direction with the most significant spin-induced effects; a good constraint on the MBH spin can still be obtained as long as the spin direction is not close to that with the least significant spin-induced effects.

Note here that the questions we addressed in this paper are based on some significant simplifications; therefore, they should be taken as the most optimistic results. In reality, there are a number of complications, such as, possible perturbation from background stars and tidal dissipation of the target stars. It has been shown that the tidal dissipation of the target stars is negligible for the parameter space studied in our paper (Psaltis et al. 2013, 2015). In the case in which the perturbation from background stars is not negligible and the orbit of the target star has some additional precession from the background stars, this precession may be modeled or removed since its evolutionary pattern is different from that of the spin-induced effects, which will be further studied in a separate work by using a hybrid relativistic model.

This work was supported in part by the National Natural Science Foundation of China under nos. 11273004, 11373031, and 11390372, and the Strategic Priority Research Program "The Emergence of Cosmological Structures" of the Chinese Academy of Sciences, Grant No. XDB09000000.

\section{REFERENCES}

Angélil, R., \& Saha, P. 2010, ApJ, 711, 157

Angélil, R., \& Saha, P. 2011, ApJ, 734, L19

Angélil, R., Saha, P., \& Merritt, D. 2010, ApJ, 720, 1303

Barker, B. M., \& O'Connell, R. F. 1975, Phys. Rev. D, 12, 329

Boyer, R. H., \& Lindquist, R. W. 1967, Journal of Mathematical Physics, 8, 265

Chandrasekhar, S. 1983, The Mathematical Theory of Black holes (Oxford: Oxford Univ. Press)

Fragile, P. C., \& Mathews, G. J. 2000, ApJ, 542, 328

Ghez, A., Salim, S., Weinberg, N. N., et al. 2008, ApJ, 689, 1044

Gillessen, S., Eisenhauer, F., Trippe, S., et al. 2009, ApJ, 692, 1075

Hairer, E., Norsett, S. P., Wanner, G. 1987, Solving Ordinary Differential Equations I. Nonstiff Problems, Springer Series in Comput. Mathematics, Vol. 8 (Springer-Verlag)

Iorio, L. 2011, MNRAS, 411, 453

Jaroszynski, M. 1998, Acta Astron. 48, 653

Kannan, R., \& Saha, P. 2009, ApJ, 690, 1553

Kerr, R. P. Physical Review Letters, 1963, 11, 237

King, A. R., \& Pringle, J. E. 2006, MNRAS, 373, L90

Lense, J., \& Thirring, H. 1918, Physikalische Zeitschrift, 19, 156

Liu, K., Wex, N., Kramer, M., Cordes, J. M., \& Lazio, T. J. W. 2012, ApJ, 747, 1

Lu, Y., \& Yu, Q. 2001, ApJ, 561, 660
Mashhoon, B., Hehl, F. W., \& Theiss, D. S. 1984, General Relativity and Gravitation, 16, 711

Merritt, D., Alexander, T., Mikkola, S., \& Will, C. M. 2010, Phys. Rev. D, 81, 062002

Meyer, L., Ghez, A. M., Schödel, R., et al. 2012, Science, 338, 84

Psaltis, D., Li, G., \& Loeb, A. 2013, ApJ, 777, 57

Psaltis, D., Wex, N., \& Kramer, M. 2016, 818, 121

Rauch, K. P., \& Blandford, R. D. 1994, ApJ, 421, 46

Rubilar, G. F., \& Eckart, A. 2001, A\&A, 374, 95

Sadeghian, L., \& Will, C. M. 2011, Classical and Quantum Gravity, 28, 225029

Stone, J. M., Eisner, J. A., Monnier, J. D., et al. 2012, ApJ, 754, 151

Wex, N., \& Kopeikin, S. M. 1999, ApJ, 514, 388

Will, C. 2008, ApJ, 674, L25

Yelda, S., Ghez, A. M., Lu, J. R., et al. 2014, ApJ, 783, 131

Yelda, S., Meyer, L., Ghez, A., \& Do, T. 2013, in Proc. Third AO4ELT Conf., ed. S. Esposito and L. Fini (Firenze: Arcetri Astrophysical Observatory), 83

Zhang, F., Lu, Y., \& Yu, Q. 2015, ApJ, 809, 127

Zucker, S., Alexander, T., Gillessen, S., Eisenhauer, F., Genzel, R. 2006, ApJ, 639, L21 